\title{
Near-Field Exposure in FM Frequencies: New Methodology and Estimation Formulas
}

\author{
Bader Mustafa Fetouri $\mathbb{D}^{1},{ }^{1 *}$ Soumaya Azzi, ${ }^{1}$ Allal Ouberehil, ${ }^{2}$ Philippe Briend, ${ }^{2}$ \\ Philippe De Doncker, ${ }^{3}$ and Joe Wiart ${ }^{1}$ \\ ${ }^{1}$ Télécom Paris, Institut Polytechnique de Paris, Palaiseau, France \\ ${ }^{2} T D F$, Rennes, France \\ ${ }^{3}$ Université Libre de Bruxelles, Brussels, Belgium
}

\begin{abstract}
Workers inside transmission pylons with FM antenna arrays are likely to be exposed to near-field radiation exceeding reference levels for occupational exposure. In this study, the near-field behavior of 64 FM pylons was studied using a new methodology. Near-field characterization was done using field metrics without taking into account field sources' size or distance from field source. The specific absorption rate (SAR) was assessed in five hundred different near-field cases using a human phantom. Estimation formulas for both local and whole-body SAR are provided and validated numerically. Local and whole-body SAR are linked to electric field strength.

(C) 2022 Bioelectromagnetics Society.
\end{abstract}

Keywords: exposure; FDTD; FM pylons; near-field; SAR

\section{INTRODUCTION}

The increasing use of wireless communication has strengthened the need for exposure monitoring, particularly near antennas where workers are likely to be exposed. The International Commission on Non-Ionizing Radiation Protection [ICNIRP, 2020] and the IEEEInternational Committee on Electromagnetic Society [IEEE, 2019] have recommended protection limits for electromagnetic field (EMF) exposure. These limits, made of basic restrictions (BRs) and reference levels (RLs), are set to avoid any health effects due to EMF exposure. BRs are the fundamental exposure limits, and were established to limit in the radiofrequency (RF) domain the specific absorption rate (SAR) that represents the absorbed power per unit mass of tissue in the human body. The BRs define the whole-body SAR (WBSAR) as well as the local SAR limits that quantify the SAR averaged over $10 \mathrm{~g}$ of tissue. BRs assessment is almost impossible in-situ. Therefore, derived limits were defined, known as reference levels (RLs) in terms of electric and magnetic field strength. The compliance to RLs ensures compliance to BRs. RLs were first defined only for WBSAR in ICNIRP [1998], and RLs for local SAR were added later in IEEE [2019] and ICNIRP [2020]. In Europe, the directive 2013/35/EU [EU, 2013], mainly based on ICNIRP [1998], requests monitoring of workers' exposure to EMFs to prevent any overexposure.

The aim of this paper is to analyze the behavior of the field near FM antennas, and to establish a relationship between the BRs and the electric field strength. The estimation of the exposure far from transmitters (known as far-field exposure) was intensively investigated using plane waves. Occupational exposure in near-field on broadcasting towers was studied in specific cases that cannot be generalized. When assessing close-to-antennas exposure, the near-field nature of EMFs depends on multiple parameters such as the physical dimensions of the EMF source, the distance from the antenna to measured fields, and the variability of EMFs along human height. A method that generalizes the nearfield human exposure assessment is proposed by this work. In order to study broadly the near-field exposure, we generated a near-field with adjustable characteristics. We studied the local and global SAR results and proposed estimation formulas that link maximum local and whole-body SAR to the electric field strength. Finding such a relationship facilitates

Grant sponsor: C2M Chair, Telecom Paris.

Conflict of interest: None.

*Correspondence to: Bader Mustafa Fetouri, Télécom Paris, Institut Polytechnique de Paris, 19 Place Marguerite Perey, F-91120 Palaiseau, France.

E-mail: b.fetouri@gmail.com

Received for review 30 September 2020; Revised 6 January 2022; Accepted 14 January 2022

DOI:10.1002/bem.22391

Published online 00 Month 2022 in Wiley Online Library (wileyonlinelibrary.com). 
the estimation of SAR in real-time in FM near-field exposure.

The studies in which EMFs are assimilated to a plane wave [Dimbylow and Gandhi, 1991; Hirata et al., 2010] investigated exposure at resonance frequencies for the human body, the variability of WBSAR due to body shape, and temperature elevation monitoring in the human body for frequencies ranging from $30 \mathrm{MHz}$ to $6 \mathrm{GHz}$. Other studies showed a plane wave's incidence angle effect on local and whole-body SAR [Conil et al., 2010], and that most of the power is absorbed by muscles for frequencies around $100 \mathrm{MHz}$ [Conil et al., 2008]. While providing insights for our study, these studies lack near-field exposure conditions. The study of plane waves in near-field is problematic when considering that in the immediate region surrounding the antenna, the angular field distribution of the antenna is distance-dependent [Vallauri et al., 2015]. Plane-wave usage suggests that the same electric field is applied on all spots of the human body surface, but in near-field the electric field is highly variable and cannot be represented by uniform plane waves. Additionally, plane waves require orthogonality between electric and magnetic fields, which is not the case in near-field [Vallauri et al., 2015]. The plane wave expansion method can describe any wave using multiple plane waves [Goodman, 2005], but was not used in this study because highly variable EMFs would need a high number of plane waves to be accurately represented, which would increase complexity.

Multiple papers studied near-field exposure to cell phones [Wiart et al., 2008; Krayni et al., 2016]. These studies were performed with mobile phone antennas for frequencies used in mobile phone communications near the head or in some other posture, hence none of the results obtained from cell phone near-field exposure can be used to assess nearfield FM exposure.

Various papers have studied workers' occupational exposure in near-field on broadcasting towers [Jokela and Puranen, 1999; Valič et al., 2012a,b]. These studies focused on worst-case scenarios where simulations exposed workers to the highest electric fields found in transmission pylons, when in reality exposure to FM antenna radiation in pylons is complex and cannot be reduced to a single worst case. Therefore, none of the above-mentioned studies generalize SAR assessment in all near-field scenarios since they all studied single cases.

A study [Findlay and Dimbylow, 2009] applied simulations to examine the impact of near-field emissions from a half-wave dipole antenna on human exposure with one emitter placed at various distances and heights. This study does not reflect the complexity of occupational environments, in which workers are exposed to EMFs from multiple sources including the reflections and diffractions caused by the metallic structure of FM masts. Another study [Gosselin et al., 2011] looked at human exposure to generic base station antennas mimicking real base stations in near-field. The paper provided estimation formulas for radiative near-field, but not for the reactive near-field due to the strong dependence of the localized absorption on the human anatomy, so the estimation formulas cannot be used to assess exposure for humans closer than $200 \mathrm{~mm}$ to base station antennas. Furthermore, this study assumed near-field conditions based on the well-known far-field formula $r \geq \frac{2 D^{2}}{\lambda}$ [Balanis, 2016], taking into account the wavelength $\lambda$, the EMF source dimension $D$, and the distance $r$ from the source to the measured field. In the case of transmission pylons, it is unknown if the source dimension should be antenna dimension or pylon dimension since the steel lattice of pylons and more generally any conductive structure constitute secondary antennas. Moreover, the far-field criteria of source dimensions and distance from source to measured field are debated and defined differently in other papers [Bansal, 1999; Laybros and Combes, 2004; Vallauri et al., 2015]. Since the nearfield nature of the environment must be taken into account when studying occupational exposure, our study proposed a method that uses field zone criteria that are independent both from distance to antenna and antenna height, and relies solely on field metrics that are defined in the methodology section.

Near-field exposure to FM antennas was studied with human models in fixed positions without taking into account the high variability of EMFs in reactive or radiating field zones. When it comes to human exposure in the literature, the usual approach is to first find the highest electric field in a given environment, and then quantify the specific absorption rate at the highest electric field position. This approach is usually followed by finding the number of cases that are over the SAR limits. In the case of occupational exposure in radio towers, this approach does not suffice because of the different exposure configurations that can be found in radio towers. The numerous near-field exposure configurations in the literature, from plane waves to real on-site full-wave exposure, express the need for a generalized exposure assessment methodology, which is the goal of this paper. This paper proposes a near-field generator based on randomly positioned dipoles, each with random intensity, phase 
shift, and tilt to recreate the near-field EMFs inside pylons. The steel lattice of FM transmission pylons causes EMFs to be reflected and currents that are induced in the lattice itself, which makes the metallic structure a sum of indirect secondary antennas; hence exposure inside pylons is made of direct and indirect reflected EMFs from FM antennas, thus justifying the near-field generation choice. The near-field EMF exposure methodology is explained first then validated using FM exposure simulations. Next, local and whole-body SAR results are shown and discussed. Finally, SAR surrogate models for workers' near-field exposure are given.

\section{NEAR-FIELD EXPOSURE ASSESSMENT: METHODOLOGY AND MATERIAL}

\section{Context and EMF Calculations for Reference}

Models of real transmission pylons with FM antennas served as reference EMFs. A near-field generator provided EMFs similar to reference EMFs. Both reference and generated EMFs were used with the near-field characterization method to find the associated field metrics, which were used to select relevant near-field volumes for finite differences in time domain (FDTD) computations for SAR evaluation. The near-field exposure assessment methodology studies EMF characteristics rather than source characteristics, thus removing the necessity of knowing antenna power, gain, and whether there is coupling between different sources.

Sixty-four entire pylons or sections of pylons, equipped with FM antennas, were modeled using EMF simulation software (Dassault Systemes, Paris, France). The pylons models were triangular, circular, or orthogonal, and they included steel lattice masts, steel ladders, platforms, and the FM antennas. Five out of 64 pylon models were validated by electric and magnetic field measurements performed inside the metallic structures. The remaining 59 pylons were generic models resembling real configurations of pylons and were studied by simulation only. Electric and magnetic fields emitted inside the pylons were computed then extracted with a 2-mm step. Our methodology studied EMF by looking at the places where workers are expected to intervene inside the pylons. This includes all EMFs inside the steel lattice, provided these EMFs are close to the emitters, so as to remain in the near-field of the emitters. Near-field conditions were verified using field metrics explained in the "Field Generation Fitting" section. The field metrics served as a base reference for the rest of our study, as they allowed us to validate the near-field exposure assessment methodology. The paper did not study exposure to the main radiation in front of antenna arrays placed on dipoles, but focused on all reachable places by workers inside pylons.

\section{Random Field GenerationThrough Multiple Dipole Combinations}

Multiple infinitesimal dipoles were used in order to recreate the highly variable near-field inside transmission pylons. The total electric and magnetic radiation of multiple dipoles was computed in a given volume. Each dipole was computed using the descriptive equations for the $\mathrm{E}$ and $\mathrm{H}$ fields [Balanis, 2016]. Each dipole had seven different inputs: amplitude, phase, two orientation angles in threedimension, and three-position coordinates $(x, y$, and $z)$. All inputs were assigned randomly in order to generate a near-field with the right characteristics. This near-field generation technique allowed as many degrees of freedom as the number of placed dipoles.

\section{Human Model, FDTD, and Homogenization}

The Duke human model was used in the standing position for the SAR computations. The phantom was reconstructed by the IT'IS foundation using detailed anatomical information from Magnetic Resonance Imaging images [Christ et al., 2009]. The FDTD method was used to solve Maxwell's equations to find electric and magnetic fields through the Yee scheme. Tissue conductivities and their relative permittivities were set for the $100 \mathrm{MHz}$ frequency. In our case, since the 2-mm grid size chosen for human exposure is small compared to the wavelength, FDTD at FM frequencies were time-consuming because of the Courant limit imposed on the time step [Courant et al., 1967]. To bypass this limitation, we rescaled the 2-mm Duke model to $4 \mathrm{~mm}$ using a tissue homogenization technique (THT) [Pradier et al., 2006], which reduced the computational cost while providing an acceptable accuracy of SAR assessment. The tissues of the human body were defined in fine grid so that the dielectric properties could be found in coarse grid. FDTD was then used to find the electric field in the coarse grid. E field values were interpolated in the fine grid so that the SAR could be calculated. In our case, THT showed an $8 \%$ error in electric field when applied at $100 \mathrm{MHz}$. Computation time was reduced from an estimated $192 \mathrm{~h}$ (8 days) to $12 \mathrm{~h}$ for one single SAR assessment using THT.

\section{Near-Field to SAR via Equivalent Principle}

In the case of a source far from the object under analysis, it is computationally costly to model the 
source and the person together using FDTD. In such a case, the equivalent principle was used to take into account the electric and magnetic fields emitted by the antenna without modeling it. The assessment of the fields induced in a given volume by a source outside of the volume was done using equivalent currents located on the surface of the volume. The equivalent principle was used to compute SAR induced by EMF in a human sized volume via the Duke human model and the well-known FDTD method. Such an equivalent principle was implemented via a "Huygens box." The near-field generator was adjusted to work with an FDTD computation machine. The generator was used to compute the Huygens box so that the FDTD calculation could be performed. The coupling between dipoles and human model was not studied in the SAR assessment as it was outside the scope of the methodology, which aimed to assess SAR to incident fields similar to the reference pylons.

\section{NEAR-FIELD CHARACTERIZATION METHOD: FIELD GENERATION FITTING}

\section{Near-Field Generation and Simulation Process}

The near-field generator provided 7 degrees of freedom per dipole, with the 8th degree of freedom being the number of dipoles. The use of several dipoles was required to generate incident near-fields similar to fields found in pylons. All dipoles were randomly placed around a human-sized volume (e.g., Fig. 1), so that dipoles were made to face the lateral sides of the human volume without being

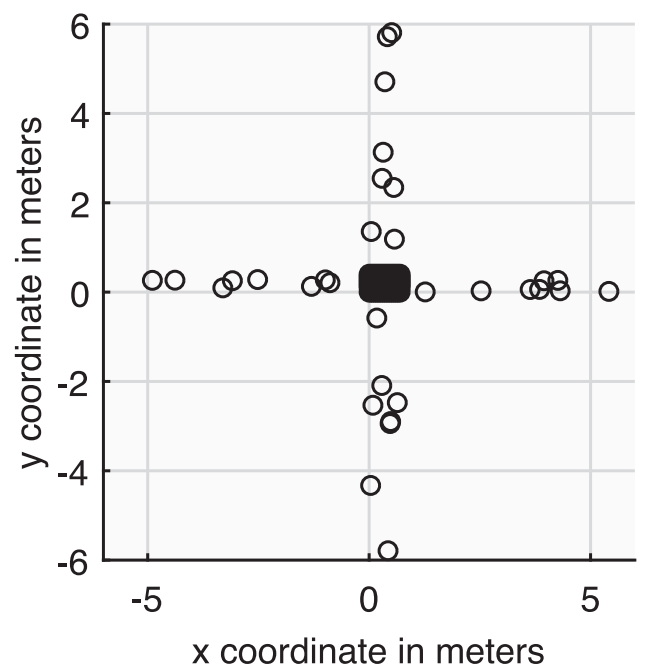

Fig. 1. Infinitesimal dipoles (empty circles) randomly placed around a human volume (filled rectangle in the middle), seen from above. Distances are in meters. inside the volume. This placement configuration yielded the best results in terms of field metrics, which are explained in the "Field metrics assessment and validation" section. In total, 32 dipoles were used, with eight dipoles facing each lateral side of the human volume. Dipoles, amplitudes, phases, and tilts were randomly assigned. The resulting EMFs from the near-field generator were found to have similar polarizations as in the pylon fields. Field metrics were then measured inside the human volume, using the EMFs that resulted from the near-field generator. Each new simulation using the near-field generator required setting dipole inputs to random values. Monte Carlo simulations were used, whereby a large number of random variables were drawn for each input, and simulations were performed millions of times until the output was accurately predicted. In our case, the near-field generator had $7 \times n_{d}$ inputs, with $n_{d}$ being the number of dipoles. For each input, 1 million variables were drawn following Latin hypercube sampling [McKay et al., 1979] to optimize the number of iterations needed to predict the output behavior. The outputs are the generated field metrics (GFM) compared to reference field metrics (RFM).

\section{Design of Experiments for Field Generation}

Since the goal is to reproduce the behavior of EMFs observed inside pylons, trials using the nearfield generator were performed following a design of experiments (DoE). The DoE tested the impact of each input of the near-field generator, as we observed the resulting behavior on field metrics. Dipole position and height coordinates are expressed in meters, amplitude is in amperes, dipole tilt and phase in radians. All values were chosen using a random uniform draw within certain boundaries. Four different scenarios called GFM 1 to GFM 4 were tested and presented in this study, where dipole tilts and phases were chosen randomly from 0 to $2 \pi$, amplitudes from 0 to $0.5 \mathrm{~A}$, and dipoles' heights ( $z$ coordinate) from 0 to $7 \mathrm{~m}$. The only varying boundaries are dipole position coordinates $x$ and $y$, which are chosen randomly from 0 to $2 \mathrm{~m}, 0$ to $4 \mathrm{~m}, 0$ to $6 \mathrm{~m}$, and 0 to $8 \mathrm{~m}$, respectively, for GFM 1 to GFM 4. GFM 1 to 4 should not be considered as overlapping scenarios. In GFM 3, dipole positions are randomly assigned on the condition that the distance between any dipole and the nearest side of the human volume is between 0 and $6 \mathrm{~m}$. All scenarios are controlled in the next section. 


\section{NEAR-FIELD CHARACTERIZATION METHOD VALIDATION}

\section{Field Metrics Assessment and Validation}

The near-field environments studied in pylons were either in the reactive or the radiative field zones. Due to the difficulties related to near-field presented in this work, field metrics for field discrimination were found and defined. Field metrics served as a mean of comparison between reference and generated fields. Four criteria were chosen for field discrimination: averaged field impedance $\frac{E}{H}$, angles formed between electric and magnetic fields $(\hat{E, H})$, field strength ratio $\frac{E_{\max }}{E_{\text {avg }}}$, and maximum electric field concentration $c(E)$. The averaged field impedance $\frac{E}{H}$ was calculated over the entire human volume, and varies from its plane-wave value $\frac{E}{H} \approx 377 \Omega$ when calculated in near-field [Vallauri et al., 2015]. The angles formed between electric and magnetic fields $(E \hat{,} H)$ vary from their plane wave value $(\hat{E, H})=\frac{\pi}{2}$ in near-field [Vallauri et al., 2015]. The field strength ratio is $\frac{E_{\max }}{E_{\text {avg }}}$, in which $E_{\max }$ is the maximum electric field spatially averaged over an $8-\mathrm{cm}$ cube so as to mimic a field probe, and $E_{a v g}$ is the electric field spatially averaged over the entire human volume with a $1-\mathrm{cm}$ step. Last is the maximum electric field concentration $c(E)$, which was defined as the distance between $E_{\max }$ and $0.9 \times E_{\max }$ in the human-sized volume. Field metrics $\frac{E}{H}$ and $(\hat{E, H})$ are related to the near-field nature of the incident fields and satisfy plane wave conditions in far-field. Field strength ratio and concentration around maximum electric field were defined in this work as field metrics for exposure assessment. All field metrics calculated in human volumes had a length of $0.4 \mathrm{~m}$ along the $x$-axis, $0.7 \mathrm{~m}$ along the $y$-axis, and $1.8 \mathrm{~m}$ along the $z$-axis, which is the volume of the phantom used for SAR assessment. Human volumes were placed in a vertical fashion to mimic the standing position of a human model. As mentioned in the "Near-field generation and simulation process" section, pylons served as reference field metrics. Human-sized volumes were slid inside each pylon, from bottom to top, side to side, thus setting the metrics in all places potentially occupied by the worker. For each field metric, a large number of values were obtained and stored as vectors. The same field metric assessment technique was applied to generated nearfields. Therefore, two sets of field metrics were obtained. Generated and reference field metrics were represented as probability density functions (PDF) to be compared. The comparison consisted of using statistical tests to see how similar the generated field metric (GFM) PDFs were when contrasted to reference field metric (RFM) PDFs. The validation of GFM and RFM distributions required the use of statistical tests [Stuart and Kendall, 1963]. Three different statistical tests were performed on GFM and RFM. The first statistical test was the histogram intersection (HI), which measured the shape differences of GFM and RFM histograms, each probed at nbins:

$$
H I=1-\frac{\sum_{1}^{n b i n s} \min \left(H_{G F M_{j}}, H_{R F M_{j}}\right)}{H_{R F M_{j}}}
$$

$H_{R F M}$ and $H_{G F M}$ are respectively GFM and RFM histograms. The second statistical test was the Hellinguer distance (HD), which was used to determine the difference between two probability distributions. Given the two probability distributions $P_{G F M}$ and $Q_{R F M}$, HD is defined as:

$$
H D\left(P_{G F M}, Q_{R F M}\right)=\frac{1}{\sqrt{2}}\left\|\sqrt{P_{G F M}}-\sqrt{Q_{R F M}}\right\|_{2}
$$

The final test was the Jensen-Shannon divergence (JSD), used to measure the behavior of two different distributions. The JSD is based on the Kullback-Leibler divergence (DKL). For two discrete probabilities $p_{G F M}$ and $q_{G F M}$, the DKL is defined as:

$$
D_{K L}\left(p_{G F M} \mid\left(q_{R F M}\right)=-\sum_{j} p(j) \log \frac{q_{R F M}(j)}{p_{G F M}(j)}\right.
$$

Let $r=\frac{p_{G F M}+q_{R F M}}{2}$, then the JSD is expressed as:

$$
\begin{aligned}
& J S D\left(p_{G F M}, q_{R F M}\right) \\
& =\frac{D_{K L}\left(p_{G F M} \|(r)+D_{K L}\left(q_{R F M} \| r\right)\right.}{2}
\end{aligned}
$$

If the HI score is under 5\%, GFM and RFM distributions were considered to have the same shape and no further statistical testing was performed. HD was used when histogram intersection did not provide a clear best performer. Last, JSD was used in case both HI and HD failed to show the best performing field metrics. The lower the results of the statistical tests, the better the performance of the GFM. The GFM with the best performance on all tests provided 
TABLE 1. GFM Performances Using Histogram Intersection (HI), Hellinger Distance (HD), and Jensen-Shannon Divergence (JSD)

\begin{tabular}{lccccc}
\hline Scenario \# & & GFM 1 & GFM 2 & GFM 3 & GFM 4 \\
\hline$E_{\text {max }} / E_{\text {avg }}$ & HI & 0.37 & 0.15 & $\mathbf{0 . 0 5}$ & 0.10 \\
& HD & $\mathrm{x}$ & $\mathrm{x}$ & $\mathrm{x}$ & $\mathrm{x}$ \\
$(E, \hat{*} H)$ & JSD & $\mathrm{x}$ & $\mathrm{x}$ & $\mathrm{x}$ & $\mathrm{x}$ \\
& $\mathrm{HI}$ & 0.08 & 0.07 & $\mathbf{0 . 0 7}$ & 0.08 \\
& $\mathrm{HD}$ & 53 & 51 & $\mathbf{4 4}$ & 56 \\
$c(E)$ & JSD & $\mathrm{x}$ & $\mathrm{x}$ & $\mathrm{x}$ & $\mathrm{x}$ \\
& HI & 0.21 & $\mathbf{0 . 1 3}$ & 0.14 & 0.13 \\
& HD & 133 & $\mathbf{8 2}$ & 88 & 86 \\
$E / H$ & JSD & $\mathrm{x}$ & $\mathrm{X}$ & $\mathrm{x}$ & $\mathrm{x}$ \\
& HI & $\mathbf{0 . 2 8}$ & 0.31 & 0.34 & 0.33 \\
& HD & $\mathbf{2 4 5}$ & 257 & 274 & 298 \\
& JSD & $\mathrm{x}$ & $\mathrm{x}$ & $\mathrm{x}$ & $\mathrm{x}$ \\
\hline
\end{tabular}

Note: $\mathrm{x}$ indicates the absence of testing necessity. Bold values indicate best score per category. Lower is better.

the best near-field generator inputs, which in turn were used to generate fields for SAR assessment following a design of experiments. Each scenario was validated using the three statistical tests (HI, HD, JSD). Table 1 shows the 4 best-performing scenarios out of hundreds of others that were tested against RFM and rejected. GFM1 and 2 performed better respectively in $\frac{E}{H}$ and $c(E)$, while GFM3 performed better at $\frac{E_{\max }}{E_{\text {avg }}}$ and $(E, \hat{,} H)$ field metrics. GFM3's best score in two out of four field metrics indicates that it is the most adapted scenario out of the four scenarios.

\section{Near-Field Choice for Exposure Assessment: Design of Experiments}

Millions of near-fields with the correct field metrics were generated. FDTD simulations for SAR assessment are time-consuming and only a limited number of near-field exposure cases were assessed with FDTD. Therefore, a DoE to maximize the diversity of cases studied was needed. Since we were able to obtain millions of near-fields, we assumed that our data set scanned all possible outcomes for the near-field generator. The generated field metrics were not uniformly distributed but followed a Gaussian-like distribution. Picking a near-field for SAR assessment using a random uniform draw could cause a discrepancy in the picks, causing highly probable near-fields to appear more in the final group draw, which in turn would cause low-probability cases to be nonexistent. This is detrimental for SAR assessment in our situation, since we would like to assess exposure with a limited number of simulations. The best SAR assessment, in this case, would look at all existing possibilities with a limited number of simulations. The solution was to perform a Gaussian to uniform transformation of the field metrics distribution using the Box-Muller transform [Box and Muller, 1958], then perform a random uniform group draw for SAR assessment.

\section{RESULTS}

\section{Local and Whole-Body SAR Results}

Using the near-field assessment methodology, 500 FDTD simulations were performed. In order to compare local SAR results, all results were normalized so that the averaged field strengths $\left(E_{\text {avg }}\right)$ in the human volume vary from 30 to $100 \mathrm{~V} / \mathrm{m}$. We define local SAR ratio $R_{10 g}$ as

$$
R_{10 g}=\frac{S A R_{10 g}}{S A R_{10 g} \text { limit }}
$$

where $S A R_{10 g}$ is the peak local SAR and $S A R_{10 g}$ limit is 10 or $20 \mathrm{~W} / \mathrm{kg}$ [ICNIRP, 2020], depending on the $S A R_{10 g}$ position. $R_{10 g} \geq 1$ indicates noncompliance, which is represented by the right side of the vertical line in Figure 2. Global exposure impact on local exposure can be better observed when $R_{10 g}$ is analyzed for different $E_{a v g}$ values. $R_{10 g}$ was calculated for different $E_{a v g}$ values then arranged in cumulative distribution functions (CDF) as shown in Figure 2. The vertical line shows the rate at which $R_{10 g}<1$, which means local SAR compliance rate. Based on the limited number of simulations that were performed, $81 \%$ of local exposure cases are compliant $\left(R_{10 g}<1\right)$ when $E_{\text {avg }}=61 \mathrm{~V} / \mathrm{m}$. The compliance rate is respectively $34 \%, 58 \%$, and $98 \%$ when $E_{\text {avg }}=100 \mathrm{~V} / \mathrm{m}$, $80 \mathrm{~V} / \mathrm{m}$, and $30 \mathrm{~V} / \mathrm{m}$. Figure 2 shows $R_{10 g}$ does not depend on $E_{\max }$ solely, as global exposure levels

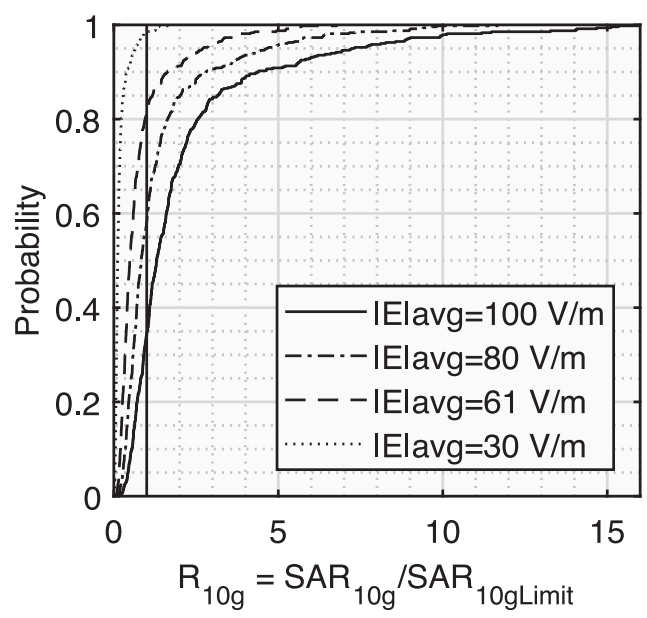

Fig. 2. Empirical cumulative distribution function of $R_{10 g}$. All results were normalized to $E_{\text {avg }}$ from 30 to $100 \mathrm{~V} / \mathrm{m}$. 


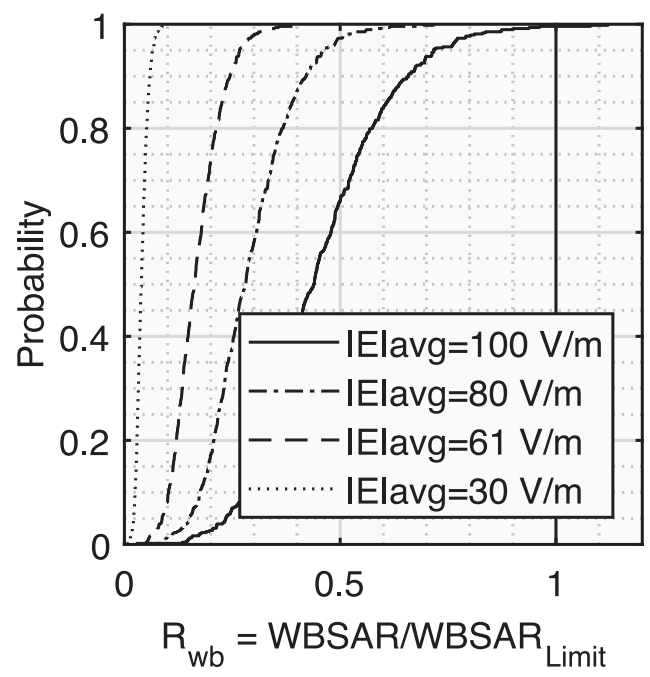

Fig. 3. $R_{w b}$ CDFs for different $E_{a v g}$ values.

represented by whole-body SAR (WBSAR) and $E_{\text {avg }}$ in the human-sized volume seem to have a major impact on compliance rates. WBSAR represents global exposure, which is best described by $E_{\text {avg }}$. Figure 3 shows the empirical CDFs of $R_{w b}$ depending on $E_{a v g} . R_{w b}$ is defined as

$$
R_{w b}=\frac{W B S A R}{W B S A R_{\text {limit }}}
$$

where WBSAR limit is $0.4 \mathrm{~W} / \mathrm{kg}$ [ICNIRP, 2020]. $R_{w b} \leq 1$ is defined as compliant WBSAR cases. $R_{w b}$ CDF is computed for different $E_{\text {avg }}$ values in Figure 3. WBSAR compliance rate is $99.6 \%$ when $E_{\text {avg }}=100$ $\mathrm{V} / \mathrm{m} E_{\text {avg }}=100 \mathrm{~V} / \mathrm{m}$. When $E_{\text {avg }} \leq 80 \mathrm{~V} / \mathrm{m}$, all simulated WBSAR cases are shown to be compliant.

Figures 2 and 3 were obtained with 500 SAR simulations, which means the cumulative distribution functions and the specified compliance rates could change if more SAR simulations were performed. Hence the uncertainty on compliance rates has to be quantified, and confidence intervals must be determined without knowing the final distribution shape. We use the bootstrapping method based on resampling [Efron and Tibshirani, 1986] to find the confidence intervals for the compliance rates. Four thousand resamples of the original 500 samples were performed, so that in total the available pool of points has two million observations. The compliance rate is calculated for both $S A R_{10 g}$ and WBSAR. We find the $R_{10 g}$ and $R_{w b}$ bootstrapping histograms from which we can derive the confidence intervals for $S A R_{10 g}$ and WBSAR results for $E_{\text {avg }}=100 \mathrm{~V} / \mathrm{m}$, which are shown in Table 2. Confidence levels shown in Table 2 correspond respectively to one to three standard deviations on the bootstrap histogram. Statistical testing using histogram intersection and Hellinger distance showed that the obtained bootstrapping histogram is a normal distribution with parameters $(\mu=0.34, \sigma=0.02)$ for $R_{10 g}$ and ( $\mu=1.00, \sigma=0.003$ ) for $R_{w b}$. The 500 observations were sufficient to give a rough estimate of compliance ratios with an uncertainty span of $10 \%$ for $R_{10 g}$ and less than $2 \%$ for $R_{w b}$, which shows that the former may need more FDTD simulations to reduce uncertainty, whereas the latter is correctly estimated with 500 observations. This phenomenon is explained by the fact that $R_{10 g}$ is more variable than $R_{w b}$.

\section{Local and Whole-Body SAR Surrogate Models}

Figure 3 suggests high correlation between WBSAR and $E_{\text {avg }}$. Based on Figure 2, it is suspected that $S A R_{10 g}$ depends on local exposure associated with $E_{\max }$ and on global exposure associated with $E_{\text {avg. }}$. Global exposure acts as a baseline for local exposure (Fig. 2), as it sets a minimum local exposure level which can only be increased by $E_{\max }$. Therefore, surrogate models for local exposure will most likely relate $S A R_{10 g}$ to both $E_{\max }$ and $E_{a v g}$, whereas the surrogate model for global exposure will link WBSAR to $E_{\text {avg. }}$. Since SAR and the Poynting vector which represents the power transfer per unit area are proportional to $E^{2}$, the squared electric field will be considered as input of the surrogate modeling. Both maximum $S A R_{10 g}$ and WBSAR are highly correlated with $E$ field as shown in Table 3 . The high correlation justifies the use of a machine learning modeling technique called linear regression [Goodfellow

TABLE 2. $\quad R_{10 g}$ and $\boldsymbol{R}_{w b}$ Confidence Intervals

\begin{tabular}{|c|c|c|c|}
\hline \multicolumn{4}{|c|}{$\boldsymbol{R}_{\mathbf{1 0 g}} \quad$ confidence intervals } \\
\hline Confidence level & $68 \%$ & $95 \%$ & $99 \%$ \\
\hline$E_{a v g}=100 \mathrm{~V} / \mathrm{m}$ & $32.9 \%-35.0 \%$ & $29.8 \%-38.3 \%$ & $28.6 \%-39.5 \%$ \\
\hline \multicolumn{4}{|c|}{$R_{w b} \quad$ confidence intervals } \\
\hline Confidence level & $68 \%$ & $95 \%$ & $99 \%$ \\
\hline$E_{a v g}=100 \mathrm{~V} / \mathrm{m}$ & $99.4 \%-99.8 \%$ & $98.9 \%-100 \%$ & $98.8 \%-100 \%$ \\
\hline
\end{tabular}


TABLE 3. Table of Correlations Between SAR and Electric Field

\begin{tabular}{lcc}
\hline Correlation & $\boldsymbol{E}_{\text {max }}^{\mathbf{2}}$ & $\boldsymbol{E}_{\text {avg }}^{\mathbf{2}}$ \\
\hline$S A R_{10 g}$ & $90.9 \%$ & $97.5 \%$ \\
$W B S A R$ & $91.2 \%$ & $98.6 \%$ \\
\hline
\end{tabular}

et al., 2016]. Linear regression requires a data set that will minimize error and maximize goodness of fit. The 500 SAR simulations will be divided into two groups: train and test [Hastie et al., 2009]. The training data set is used to determine the regression coefficients, whereas the test data set is used to calculate the $R^{2}$ value, which will determine the goodness of the fit [Draper and Smith, 2014]: $R^{2}=1-\frac{\sum_{i=1}^{n}\left(y_{i}-\hat{y}_{i}\right)^{2}}{\sum_{i=1}^{n}\left(y_{i}-\bar{y}\right)^{2}}$ where $n$ is the number of samples, $y_{i}$ is the sample $i$, $\hat{y}_{i}$ is the predicted value, and $\bar{y}$ the average of all samples. Ten thousand regressions are computed with randomly chosen data points for both test and train groups. The chosen points are different in each simulation, each giving a new $R^{2}$ value. The metric allowing discrimination is averaged $R^{2}$. Since $S A R_{10 g}$ has different limits based on its peak localization being in the head and trunk or limbs, it is appropriate to find a surrogate model for each. Thus all data are divided into head and trunk and limbs, which are respectively $43 \%$ and $57 \%$ of all $S A R_{10 g}$ localizations. Best $R^{2}$ performance shows that the most accurate surrogate model is given by 70 and 90 training data points respectively for $S A R_{10 g}$ localized in head and trunk and limbs. The $S A R_{10 g}$ regression model shows that basic restriction levels for head and trunk and limbs are exceeded respectively when $E_{\max }>187 \mathrm{~V} / \mathrm{m}$ and $E_{\max }>310 \mathrm{~V} / \mathrm{m}$, assuming that $E_{\text {avg }}=61 \mathrm{~V} / \mathrm{m}$ in both cases. $S A R_{10 g}$ is written $S A R_{10 g}=\beta_{0} \times E_{\text {avg }}^{2}+\beta_{1} E_{\max }^{2}$ with $\beta$ coefficients provided in Table 4 . Best $R^{2}$ performance shows the most accurate surrogate model for WBSAR is given by 160 training points. The regression model shows that WBSAR limit of $0.4 \mathrm{~W} / \mathrm{kg}$ is reached when $E_{\text {avg }}>122 \mathrm{~V} / \mathrm{m}$. WBSAR is described by

$$
W B S A R=2.7 \times 10^{-5} \times E_{a v g}^{2}
$$

TABLE 4. Table of $\beta$ Coefficients (in $S \cdot \mathrm{m}^{2} \cdot \mathrm{kg}^{-1}$ ) for $S A R_{10 g}$ Surrogate Models

\begin{tabular}{lcc}
\hline Coefficients $\left(\mathbf{S} \cdot \mathbf{m}^{\mathbf{2}} \cdot \mathbf{k g}^{\mathbf{- 1}}\right)$ & $\boldsymbol{\beta}_{\mathbf{0}}$ & $\boldsymbol{\beta}_{\mathbf{1}}$ \\
\hline Head \& Trunk & $2.0 \times 10^{-3}$ & $7.3 \times 10^{-5}$ \\
Limbs & $1.9 \times 10^{-3}$ & $1.3 \times 10^{-4}$ \\
\hline
\end{tabular}

WBSAR surrogate model shows a higher reference value than actual simulations in Figure 3 where it was shown that $E_{\text {avg }}=100 \mathrm{~V} / \mathrm{m}$ causes a compliance rate of $99 \%$. Since SAR in the human body is impossible to measure, the results of WBSAR and $S A R_{10 g}$ from the estimation formulas were validated by comparison to the numerical FDTD results.

\section{Surrogate Model Deviation from SAR Simulations}

The histograms of the deviation of the estimation formulas from the numerical FDTD simulations are presented in Figure 4. The WBSAR and $S A R_{10 g}$ results are displayed as a ratio to the SAR limit such as:

$$
\text { Deviation }(d B)=10 \times \log _{10}\left(\frac{S A R_{\text {model }}}{S A R_{\text {sim }}}\right)
$$

where $S A R_{\text {model }}$ is the WBSAR or $S A R_{10 g}$ value obtained using the estimation formulas, and $S A R_{\text {sim }}$ is the WBSAR or $S A R_{10 g}$ value obtained through FDTD simulations. In Figure 4, positive deviations mean that $S A R_{\text {model }}$ is conservative when compared to numerical FDTD simulations $S A R_{\text {sim }}$, whereas negative deviations mean that estimation formulas are not conservative.

It can be observed in Figure 4 that estimation formulas are not conservative in all cases. Based on 500 SAR assessments using the methodology described in this paper, estimation formulas are conservative in $91 \%, 63 \%$, and $70 \%$ of cases respectively for WBSAR, head and trunk $S A R_{10 g}$, and limb $S A R_{10 g}$ models. Since the conservativeness of estimation formulas was not an objective in this work, it is no surprise that conservative percentages are under $100 \%$. Other papers built surrogate models [Gosselin et al., 2011] using a 95th percentile cuboid, which is a human body leading to a conservative exposure for $95 \%$ of the population. Figure 4 also shows the difference between surrogate models and actual SAR observations for the same inputs.

\section{DISCUSSION}

\section{Surrogate Models' Versatility}

Although the SAR and WBSAR surrogate models were obtained using FDTD simulations at $100 \mathrm{MHz}$, they are valid for all FM frequencies with a maximum error of 5\% for the Duke human model. In fact, SAR is frequency-dependent due to the human tissue conductivities, which can change, at the most, by $5 \%$ in the FM frequency range. To ensure their 

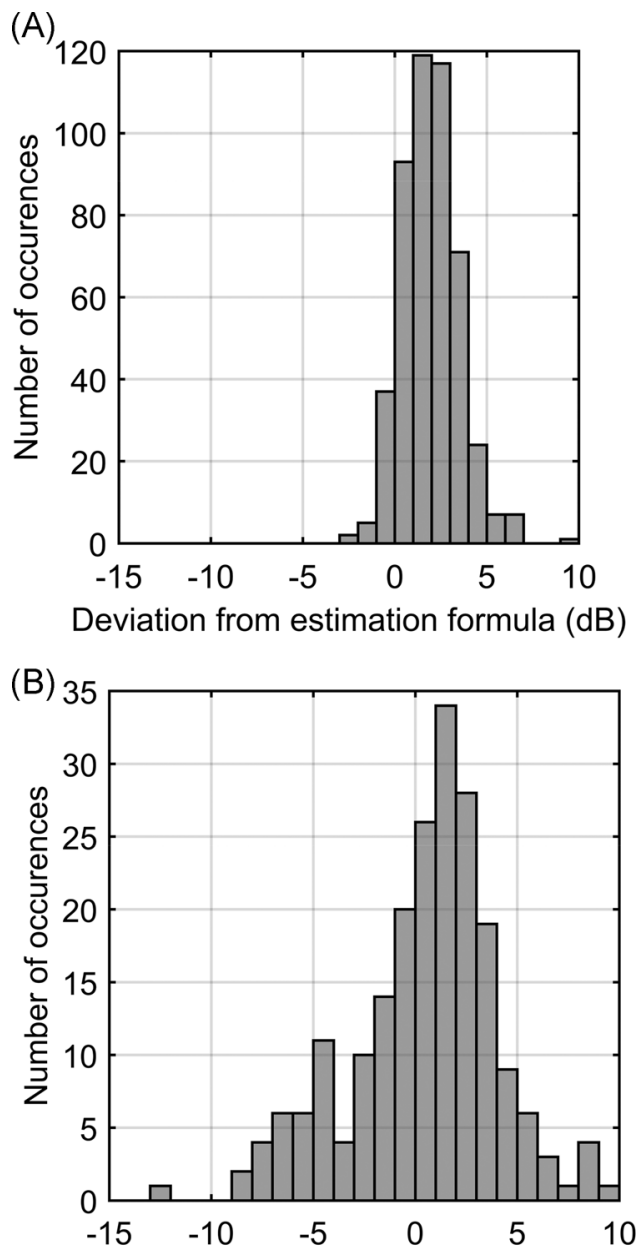

Deviation from estimation formula $(\mathrm{dB})$

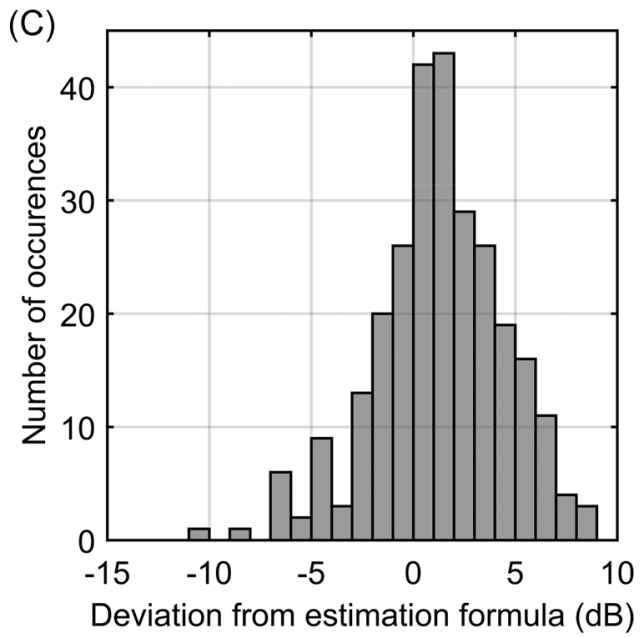

Fig. 4. Histograms of the deviations between simulation results and estimation formulas. (A) WBSAR, (B) head and trunk $S A R_{10 g}$, and (C) limbs $S A R_{10 g}$. precision, the surrogate models were built using $E_{\text {avg }}$ sampled at a $1-\mathrm{cm}$ step. However, they can be used with $E_{\text {avg }}$ measurements performed at other grid steps, as references [Larchêveque et al., 2005; IEC, 2017] show that electric field averaging using a limited number of points is valid with an error.

\section{Surrogate Models' Limitations}

The surrogate models presented in this paper were found using linear regression, a statistical method for examining the relationship between a dependent output and one or more independent inputs. Hence, the accuracy of the obtained surrogate models depends on the number of SAR simulations. The linear regression models were trained in order to avoid over-fitting. But linear regression remains sensitive to outliers, which can cause models to hide the relationship between variables. Other methods such as Polynomial Chaos Expansion and Kriging were looked at when performing the study. Due to different maximum field strengths causing the same peak local SAR values, both surrogate modeling methods could not be used.

\section{Uncertainty of Results}

In order to bypass limitations imposed by the size of the FDTD computations in FM frequencies, the human model was homogenized with an $8 \%$ error on the electric field strength results and a $16 \%$ error on local and whole-body SAR results. The uncertainty quantification using bootstrapping methods exposed the confidence intervals at different confidence levels. The local and whole-body surrogate models in this paper are approximations with their deviation from reality studied and presented in Figure 4. It is important to be aware that any conclusion made based on the results in this paper must take into account all uncertainties, and that the surrogate models for local and whole-body SAR are mere approximations.

\section{Expansion to Other Frequencies}

Both the radio tower simulations and the SAR calculations were performed at $100 \mathrm{MHz}$, but the method itself can be applied to frequencies where dosimetric reference levels can be used as an exposure limit for local exposure (typically from $100 \mathrm{kHz}$ to $6 \mathrm{GHz}$, according to IEEE [2019]), on the condition that validated reference simulations are used for the 
reference field metrics, and that the generated field metrics are validated using the method described in this paper.

\section{CONCLUSION}

This work presented a new method for exposure assessment in near-field environments by focusing on the electric and magnetic fields rather than sources. This method depends on a chain in which there is a near-field generation and characterization, statistical testing for near-field validation, local and whole-body SAR simulations via FDTD, and exposure assessment substitute formulas using linear regression. An application in near-field FM exposure was presented, using 500 local and whole-body SAR simulations, but the methodology could have been applied for different frequencies and sources. Due to local SAR simulations showing a dependency on both maximum and averaged field strength in a human-sized volume, local SAR estimation formulas are provided with these two quantities. The whole-body SAR estimation formula only uses the averaged electric field strength in a human-sized volume. Both SAR quantities were linked to electric fields that can be measured on site using a field-meter. Future works may include using the near-field exposure method in other frequencies with the 95th percentile cuboid to find estimation formulas leading to a conservative SAR.

\section{REFERENCES}

Balanis CA. 2016. Antenna Theory: Analysis and Design. Hoboken, NJ: John Wiley \& Sons. pp. 27-154.

Bansal R. 1999. The far-field: How far is far enough? Appl Microw Wirel 11:58-60.

Box GEP, Muller ME. 1958. A note on the generation of random normal deviates. Ann Math Stat 29:610-611.

Christ A, Kainz W, Hahn EG, Honegger K, Zefferer M, Neufeld E, Rascher W, Janka R, Bautz W, Chen J. 2009. The Virtual Family_Development of surface-based anatomical models of two adults and two children for dosimetric simulations. Phys Med Biol 55:N23-38.

Conil E, Hadjem A, Lacroux F, Wong MF, Wiart J. 2008. Variability analysis of SAR from $20 \mathrm{MHz}$ to $2.4 \mathrm{GHz}$ for different adult and child models using finite-difference time-domain. Phys Med Biol 53:1511-1525.

Conil E, Hadjem A, Gati A, Wong M-F, Wiart J. 2010. Influence of plane-wave incidence angle on whole body and local exposure at $2100 \mathrm{MHz}$. IEEE Trans Electromagn Compat 53:48-52.

Courant R, Friedrichs K, Lewy H. 1967. On the partial difference equations of mathematical physics. IBM J Res Dev 11:215-234.

Dimbylow PJ, Gandhi OP. 1991. Finite-difference time-domain calculations of SAR in a realistic heterogeneous model of the head for plane-wave exposure from $600 \mathrm{MHz}$ to $3 \mathrm{GHz}$. Phys Med Biol 36:1075-1089.

Draper NR, Smith H. 2014. Applied regression analysis, 326. Hoboken, NJ: John Wiley \& Sons. pp. 135-149.

Efron B, Tibshirani R. 1986. Bootstrap methods for standard errors, confidence intervals, and other measures of statistical accuracy. Stat Sci 1:54-75.

EU. 2013. Directive 2013/35/EU of the European parliament and of the council of 26 June 2013 on the minimum health and safety requirements regarding the exposure of workers to the risks arising from physical agents (electromagnetic fields) (20th individual Directive within the meaning of Article 16(1) of Directive 89/391/EEC) and repealing Directive 2004/40/EC). Off J Eur Union 2013:179/ $1-179 / 21$.

Findlay RP, Dimbylow PJ. 2009. Spatial averaging of fields from half-wave dipole antennas and corresponding SAR calculations in the NORMAN human voxel model between 65 MHz and 2 GHz. Phys Med Biol 54:2437-2447.

Goodfellow I, Bengio Y, Courville A. 2016. Deep Learning. Cambridge, MA: MIT Press. pp. 98-120.

Goodman JW. 2005. Introduction to Fourier optics. Englewood, Colorado: Roberts and Company Publishers. pp. 55-61.

Gosselin MC, Vermeeren G, Kühn S, Kellerman V, Benkler S, Uusitupa TMI, Joseph W, Gati A, Wiart J, Meyer FJC, Martens L, Nojima T, Hikage T, Balzano Q, Christ A, Kuster N. 2011. Estimation formulas for the specific absorption rate in humans exposed to base-station antennas. IEEE Trans Electromagn Compat 53:909-922.

Hastie T, Tibshirani R, Friedman J. 2009. The Elements of Statistical Learning: Data Mining, Inference, and Prediction. New York, NY: Springer Science \& Business Media. pp. 43-57.

Hirata A, Fujiwara O, Nagaoka T, Watanabe S. 2010. Estimation of whole-body average SAR in human models due to planewave exposure at resonance frequency. IEEE Trans Electromagn Compat 52:41-48.

ICNIRP. 1998. Guidelines for limiting exposure to time-varying electric, magnetic, and electromagnetic fields (up to 300 GHz). Health Phys 74:494-521.

ICNIRP. 2020. Guidelines for limiting exposure to electromagnetic fields (100 kHz to $300 \mathrm{GHz}$ ). Health Phys 118:483-524.

IEC. 2017. Determination of RF field strength and SAR in the vincinity of radiocommunication base stations for the purpose of evaluating human exposure. IEC 62232:2017.

IEEE. 2019. IEEE standard for safety levels with respect to human exposure to radio frequency electromagnetic fields, $3 \mathrm{kHz}$ to 300GHz. IEEE C951-2019.

Jokela K, Puranen L. 1999. Occupational RF exposures. Radiat Prot Dosimetry 83:119-124.

Krayni A, Hadjem A, Sibille A, Roblin C, Wiart J. 2016. A novel methodology to evaluate uplink exposure by personal devices in wireless networks. IEEE Trans Electromagn Compat 58:896-906.

Larchêveque E, Dale C, Wong MF, Wiart J. 2005. Analysis of electric field averaging for in situ radiofrequency exposure assessment. IEEE Trans Veh Technol 54:1245-1250.

Laybros S, Combes PF. 2004. On radiating-zone boundaries of short, $\$ \lambda / 2 \$$, and $\$ \lambda \$$ dipoles. IEEE Antennas Propag Mag 46:53-64.

McKay MD, Beckman RJ, Conover WJ. 1979. Comparison of three methods for selecting values of input variables in the analysis of output from a computer code. Technometrics 21:239-245. 
Pradier A, Lacroux F, Lautru D, Wong MF, Hanna VF, Wiart J 2006. SAR assessment using tissues homogenization technique. In: Eur Sp Agency (Special Publ ESA SP Vol. 626) SP, pp. 1-4.

Stuart A, Kendall MG. 1963. The Advanced Theory of Statistics. London: Charles Griffin \& Company. pp. 161-186.

Valič B, Gajšek P, Kos B. 2012a. Occupational exposure assessment on an fm mast: Electric field and sar values. Int J Occup Saf Ergon 18:149-159.
Valič B, Kos B, Gajšek P. 2012b. Simultaneous occupational exposure to FM and UHF transmitters. Int J Occup Saf Ergon 18:161-170.

Vallauri R, Bertin G, Piovano B, Gianola P. 2015. Electromagnetic field zones around an antenna for human exposure assessment: Evaluation of the human exposure to EMFs. IEEE Antennas Propag Mag 57:53-63.

Wiart J, Hadjem A, Wong MF, Bloch I. 2008. Analysis of RF exposure in the head tissues of children and adults. Phys Med Biol 53:3681-3695. 